\title{
NO ASSOCIATION BETWEEN GRIP STRENGTH AND CARDIOVASCULAR RISK; THE COLAUS POPULATION-BASED STUDY
}

\author{
Cédric Gubelmann ${ }^{1}$; Peter Vollenweider ${ }^{1}$; Pedro Marques-Vidal ${ }^{1}$ \\ Department of Medicine, Internal Medicine, Lausanne University Hospital, Switzerland
}

Authors' emails:

Cédric Gubelmann: Cedric.Gubelmann@chuv.ch

Peter Vollenweider: $\quad$ Peter.Vollenweider@chuv.ch

Pedro Marques-Vidal : $\quad$ Pedro-Manuel.Marques-Vidal@chuv.ch

Address for correspondence and reprints:

Cédric Gubelmann

CoLaus Study (BU19_02 627)

Lausanne University Hospital

Rue du Bugnon 19

1011 Lausanne

Switzerland

Phone : +41213140346

Email : Cedric.Gubelmann@chuv.ch

The authors report no conflict of interest.

Word count abstract: 1912

Number of tables: $z 3 \quad$ Figures: 1
Main text: 23282653

References: 2330 


\section{ABSTRACT}

Background: Decreased grip strength (GS) is predictive of cardiovascular (CV) disease but whether it improves CV risk prediction has not been evaluated. We assessed the predictive value of low GS on incident CV events and overall mortality taking into account CV risk equations in a population-based study from Switzerland.

Methods: 2707 adults (54.8\% women, age range 50-75 years) were followed for a median time of 5.4 years. GS was assessed using a hydraulic hand dynamometer. CV absolute risk at baseline was assessed using recalibrated SCORE, Framingham and PROCAM risk equations. Incident CV events were adjudicated by an independent committee.

Results: 160 deaths and 188 incident CV events occurred during follow-up. On bivariate analysis, low GS was associated with increased incident CV events: Hazard Ratio (HR) and (95\% confidence interval) $1.76(1.13-2.76), p<0.01$ but not with overall mortality: HR=1.51 (0.942.45), $p=0.09$. The association between low GS and incident CV events disappeared after adjusting for baseline CV risk: $H R=1.23(0.79-1.94), p=0.36 ; 1.34(0.86-2.10), p=0.20$ and 1.47 (0.94-2.31), $p=0.09$ after adjusting for SCORE, Framingham and PROCAM scores, respectively.

Conclusion: Low GS is not predictive of incident CV events when taking into account CV absolute risk.

Abstract word count: 1912

Keywords: grip strength; CV events incidence; cardiovascular risk assessment; Switzerland; population-based study; adults. 


\section{INTRODUCTION}

Grip strength (GS) has been shown to be inversely associated with risk of incident cardiovascular (CV) events $(1,2)$ and overall mortality $(1,3)$. The effect of low GS on CV events might be partly mediated by changes in CV risk factors (4); thus, the analysis of the effect of low GS on CV events and overall mortality should take into account basal CV risk. Basal CV risk can be estimated using equations such as SCORE (5), Framingham (6) and PROCAM (7). Although the associations of GS with incident CV events $(1,2)$ and overall mortality $(1,3,8)$ have been assessed in several longitudinal studies, they were only partially adjusted on CV risk factors. Finally, whether low GS improves the predictive value of the existing CV risk equations remains to be assessed.

Thus, the aim of this study was to assess the predictive value of low GS on CV events incidence and overall mortality, taking into account absolute CV risk at baseline as assessed by SCORE, Framingham or PROCAM equations, in a well-characterised population-based sample from the city of Lausanne, Switzerland (CoLaus study).

\section{MATERIALS AND METHODS}

\section{Recruitment}

The detailed description of the recruitment of the CoLaus study has been published previously (9). Briefly, the CoLaus study is a population-based cohort exploring the biological, genetic and environment determinants of CV diseases. A non-stratified, representative sample of the population of Lausanne (Switzerland) was recruited between 2003 and 2006 based on the following inclusion criteria: a) age 35-75 years and b) willingness to participate. The baseline 
sample included 6733 participants. Participants aged over 50 years (3704 of the 6733 initially recruited, 55\%) were invited to participate in a sub-study on frailty, which included GS assessment.

Grip strength

All participants aged over $50(\mathrm{~N}=3704)$ were invited to participate in a sub-study on frailty, which included GS. GS was assessed using the Baseline ${ }^{\circledR}$ Hydraulic Hand Dynamometer and positioning of the participants was done according to the American Society of Hand Therapists's guidelines (10): subject seated, shoulders adducted and neutrally rotated, elbow flexed at $90^{\circ}$, forearm in neutral position and wrist between 0 and $30^{\circ}$ of dorsiflexion. Three measurements were performed consecutively with the right hand. Coefficient of variation between measurements was $5.3 \%$. The highest value (expressed in $\mathrm{kg}$ ) was included in the analyses. Participants were also asked about their handedness. Grip strength was categorized as low or normal according to Fried criterion (11) that takes into account gender and body mass index.

\section{Clinical data}

Socio-demographic data such as maritalstatus, education level, job position and social help, together with tobacco, leisure-time and occupational physical activity data were collected by questionnaire. Participants were considered as physically active if they practiced a leisuretime physical activity of at least two periods of $\geq 20$ minutes per week. Leisure-time physical activity was categorized as $<2$ or $\geq 2$ periods of $\geq 20$ minutes per week. Occupational activity was categorized as non-physical (when sitting or standing) and physical (carrying light or heavy 
load). Personal and family history of CV disease and CV risk factors was elicited with a standardized interview questionnaire filled in by a trained recruiter. Participants also indicated if they were treated for hypertension, dyslipidemia or diabetes.

Body weight and height were measured to the nearest $0.1 \mathrm{~kg}$ and $5 \mathrm{~mm}$, respectively, using a Seca ${ }^{\circledR}$ scale and height gauge (Hamburg, Germany), with participants in light indoor clothes standing without shoes. Waist and hip circumferences were measured as recommended (12) at mid-way between the lowest rib and the iliac crest, and at the greater trochanters, respectively. Blood pressure (BP) was measured using an Omron ${ }^{\circledR}$ HEM-907 automated oscillometric sphygmomanometer (13) after at least 10 minutes' rest in a seated position and the average of the last two measurements was used. Hypertension was defined as a systolic BP $\geq 140 \mathrm{mmHg}$ and/or a diastolic BP $\geq 90 \mathrm{mmHg}$ and/or presence of an anti-hypertensive treatment.

\section{Biological data}

A fasting venous blood sample was drawn and measurements performed by the clinical laboratory of the Lausanne university hospital. CV risk factors included glucose, total and HDLcholesterol, triglycerides; LDL-cholesterol was calculated using the Friedewald formula if triglycerides were $<4.6 \mathrm{mmol} / \mathrm{L}$. Diabetes was defined by a fasting glucose $\geq 7.0$ and/or presence of antidiabetic drug treatment. Dyslipidemia was defined either by the presence of a hypolipidemic drug or using the LDL-cholesterol thresholds according to the PROCAM CV score (7) adapted for Switzerland (14). 


\section{Cardiovascular risk assessment}

$\mathrm{CV}$ risk was calculated using internationally used risk equations. As there is no consensus regarding which risk equation to use in Switzerland (15), we opted for the three most used equations: the European Society of Cardiology SCORE (5), Framingham-2001 (6) and PROCAM2007 (7). Framingham-2001 and SCORE have been recalibrated $(16,17)$ and validated on the Swiss population $(17,18)$. The SCORE, Framingham 2001 and PROCAM 2007 risk equations use age, gender, parental history, smoking, blood pressure, lipids and diabetes data to compute the 10-year absolute risk of CV death, coronary heart disease (CHD) and CV events, respectively. Participants were categorized as low, medium, high or very high CV risk according to cutoffs shown in Supplementary Table 1. Participants with previous history of CV disease were considered at very high CV risk.

\section{Outcomes}

Outcomes of interest were CV events and overall deaths. CV events included cerebrovascular events (CBV) and CHD. CBV events were defined as transient ischemic attack, ischemic or hemorrhagic stroke, amaurosis fugax and transient global amnesia. CHD events were defined as myocardial infarction, stable or unstable angina, coronary revascularization or bypass grafting. Outcomes were first verified and medically documented by a trained investigator, and further validated using pre-defined criteria by an independent adjudication committee composed of internists, cardiologists and a neurologist. 


\section{Exclusion criteria}

Participants were excluded if they presented a questionable GS fi.e. any condition precluding adequate measurement of GS such as pain, injury, recent surgery, osteoarthritis and theumatoid arthritis, among others), or if no follow-up data were available. Questionable GS values were considered if the participant reported any condition precluding adequate measurement (i.e. pain, injury, recent surgery, osteoarthritis and rheumatoid arthritis, among others), irrespectively of the observed value.

\section{Statistical analysis}

Statistical analyses were conducted using Stata version 14.0 for windows (Stata Corp, College Station, Texas, USA). Descriptive analyses were expressed as number of participants (percentage) for categorical variables or as average \pm standard deviation for continuous variables. Between-group comparisons were performed using chi-square and Student t-test for categorical and continuous variables, respectively.

The effect of low GS on incident CV events and overall mortality was assessed using Cox proportional hazards models and results were expressed as hazard ratio (HR) and 95\% confidence interval $(95 \% \mathrm{Cl})$. Bivariate and multivariate analyses were performed, and the following multivariate models were used: 1) adjusted on age and gender; ; 2) age, gender, education level, job position and social help; 3) age, gender, education level, job position, social help, waist-to-hip ratio and height; 4) adjusted on absolute CV risk according to SCORE; 5) adjusted on absolute CV risk according to Framingham 2001, and 6) adjusted on absolute CV risk according to PROCAM 2007. Adjustments on CV risk factors' treatment were also performed. To take into account the decline in muscular performance occurring with age, 
sensitivity analyses were performed by further stratifying on tertiles of age. Further adjustments on socioeconomic markers were also performed. Statistical significance was assessed for a two-sided test with $\mathrm{p}<0.05$.

Power analysis was conducted using the power cox function of Stata. The following parameters were calculated: 1) power to consider the observed HR as statistically significant at $p=0.05 ; 2$ ) the minimum sample size to consider the observed HR as statistically significant at a power of 0.80 and $p=0.05$, and 3 ) the minimum detectable HR taking into account a sample size of 2707,160 deaths and 188 incident CV events, a power of 0.80 and $p=0.05$. Power analyses were not performed if the observed HR was less than 1.

\section{Ethical statement}

The Colaus study was approved by the Ethics Committee of the University of Lausanne and The institutional Ethics Committee of the University of Lausanne (19) approved the baseline CoLaus study (protocol reference $16 / 03$, decisions of $13^{\text {th }}$ January and $10^{\text {th }}$ February 2003) and the approval was renewed for its follow-up (protocol reference 33/09, decision of $23^{\text {rd }}$ February 2009). aAll participants gave their signed informed consent before entering the study.

\section{RESULTS}

Characteristics of included and excluded participants

The selection procedure is indicated in Figure 1. Of the initial 3704 participants aged 50 and over, 2707 (73.1\%) were retained for analysis. The characteristics of the included and excluded participants are summarized in Supplementary Table 2. Included participants were 
more likely right-handed, physically active and to perform leisure-time physical activity, more educated, had a higher job position and were less prone to smoke, to receive social help, to present with hypertension or dyslipidemia than excluded ones. No association was found in absolute CV risk using SCORE and Framingham risk equations, whereas excluded participants had slightly higher CV risk according to the PROCAM risk equation.

Participants' characteristics overall and according to GS category are summarized in Table 1. Participants with a low GS were older, less likely to have a high education level, working or to performing leisure-time physical activity physically active. Participants with a low GS were also more likely to receive social help and had a higher baseline absolute CV risk. GS values according to gender are represented in Supplementary Figure 1. Mean \pm standard deviation GS were $26.1 \pm 5.3 \mathrm{~kg}$ for women and $42.7 \pm 8.4 \mathrm{~kg}$ for men.

Association of grip strength with outcomes

During a median follow-up time of 5.4 years, there were 160 deaths and 188 incident CV events. Survival curves for all causes and CV events according to GS category are shown in Supplementary Figure 2. Five-year overall survival was $96.9 \%$ (95\% confidence interval: 96.1 97.5) and 93.5\% (88.9-96.3) for normal and low GS (P value: 0.09), respectively. Five-year CV events-free survival was $95.5 \%(94.6-96.3)$ and $89.0 \%(83.4-92.7)$ for normal and low GS (P value: 0.01$)$, respectively.

The unadjusted and multivariate-adjusted associations between low GS and overall mortality or incident CV events are described in Table 2. Unadjusted analyses showed that low GS was associated with a higher incidence of CV events, while no association was found with 
overall mortality. The association between low GS and incident CV events was no longer significant after multivariate adjustment (Table 2). Results did not change after adjustment on CV risk factors' treatment (Supplementary Table 3) or after stratification by tertiles of age (Supplementary Tables 4 and 5).

\section{Discussion}

This study assessed the impact of low GS on overall mortality and incident CV events in a prospective, population-based sample with a median 5.4-year follow-up time. Our results suggest that the association between low GS and incident CV events is no longer significant after adjusting for baseline absolute CV risk. Thus, GS measurement does not seem to be useful in assessing CV risk beyond traditional CV risk estimation equations.

\section{Grip strength and incident cardiovascular events}

Low GS was significantly associated with an increase in incident CV events on bivariate analysis, but this association disappeared after multivariate adjustment. These findings are in agreement with the study by Fujita et al. in from Japan (20). However our results differ from

those of the PURE study (1). It has to me be mentioned that in the latter study, GS was reported as 5-kg decrease and not dichotomized in low and normal, and furthermore CV risk factors were self-reported. Discrepancies could therefore possibility result from those methodological aspects. Other longer follow-ups studies $(2,3,21,22)$ also showed an inverse association between different markers of GS (i.e. standard deviation, deciles or tertiles) and incident CV events, after adjustment on a small number of CV risk factors. Thus, several studies have shown an inverse association between GS and incident CV disease events, but the results are difficult to apply in a clinical setting as different metrics for GS have been used and no threshold below 
which the CV risk can be considered as increased was suggested. Similarly, although several studies $(1,22)$ adjusted the results for gender, this adjustment might not have cancelled out the considerable difference in GS levels between genders. In this study, we assessed whether a common definition of low GS was associated with incident CV events. Our results suggest that the effect of low GS on incident CV events is mediated by CV risk factors, as the association disappears after adjusting for absolute CV risk. Still, it would be of interest to replicate our study in other population-based samples, in order to confirm or infirm if a low GS is associated with incident CV events independently of the other CV risk factors.

\section{Grip strength and overall mortality}

Low GS was associated with overall mortality neither on bivariate, nor on multivariate analysis. These findings are partially in agreement with two studies $(20,22)$ showing similar results for women though not for men but it has been contradicted by other studies $(1,3,8,21)$ showing that different markers of GS were negatively associated with overall mortality. A possible explanation might be the relatively short follow-up time in our sample, or the fact that we adjusted for absolute CV risk while the other studies only adjusted on self-reported (1) or on a limited number of CV risk factors $(3,8,21)$. Overall, our results suggest that low GS has no impact on overall mortality when absolute CV risk is taken into account.

\section{Study limitations}

This study has several limitations worth acknowledging. Firstly, GS was assessed on the right hand whereas approximately $7 \%$ of our participants were left-handed. Although the use of the non-dominant hand might lead to lower GS values, most studies reported no difference (2325), while some reported slightly higher values for the dominant compared to the non- 
dominant hand $(26,27)$. Thus, GS measurement at the right hand irrespective of handedness will have a limited impact on the observed values. Hover, it has been sh not differ between dominant and non-dominant hands in left-handed subjects (10). Secondly, the exclusion of questionable GS was based on self-reported information given by the participant (i.e. condition that may preclude adequate measurement), and did not rely on objective criteria. However, including all GS measurements led to similar conclusions for overall mortality and partially for incident CV events, for which small significant positive associations $(p<0.05)$ were found after adjustment for Framingham or PROCAM risk equations (see Supplementary Table 6). Still, the p-values would not resist Bonferroni correction, and the PROCAM risk equation hasn't been validated for the Swiss population. Thirdly, some events such as amaurosis fugax (AF) and transient global amnesia (TGA) might be wrongly reported as CV. Still, in this study, AF (N=1) and TGA (N=4) represented only $2.7 \%$ of $C V$ events, so that the impact of a possible ascertainment bias is low. Further, excluding AF and TGA events led to similar conclusions (see Supplementary Table 7). Fourthlys our sample size and followup time period are relatively small for our low-risk population, leading to a relatively low statisticalpower; However, on the whole sample, power sample calculations showed that the overall power to consider the bivariate and multivariate-adjusted HR as significant was higher than $70 \%$ in most all but one cases (Supplementary Table 3). The ongoing follow-up of the CoLaus study will enable assessing the 10-year outcomes of the participants. Fifthly, one-fifth of the participants did not participate to follow-up, but this participation rate is comparable to the literature (5), and loss to follow-up has only limited impact on relative risks for exposure-risk associations (28). SixthlyThirdly, our data have been collected between 2003 and 2012, 
whereas some previous findings' data were collected before $2000(2,22,29)$. At this time, the incidence of fatal CV events was higher (30), which might have allowed to demonstrate the association between GS and incident CV events. FinallyFourthly, only participants aged between 50 and 75 were included, so our findings cannot be extrapolated to other ages.

\section{Conclusion}

In a prospective, population-based sample aged 50 to 75 years, low GS was associated neither with overall mortality nor with incident $\mathrm{CV}$ events when adjusting for absolute $\mathrm{CV}$ risk.

\section{FUNDING}

The CoLaus study was and is supported by research grants from GlaxoSmithKline, the Faculty of Biology and Medicine of Lausanne, and the Swiss National Science Foundation (grants 3200B0-105993, 3200B0-118308, 33CSCO-122661, 33CS30-139468 and 33CS30-148401). The funding sources had no role in study design; in the collection, analysis and interpretation of data; in the writing of the report; and in the decision to submit the article for publication.

\section{AUTHORS' CONTRIBUTIONS}

CG made most of the statistical analyses and wrote most of the article; PMV made part of the statistical analysis and wrote part of the article; PV revised the article for important intellectual content.

\section{CONFLiCt OF INTEREST}

The authors report no conflict of interest. 


\section{REFERENCES}

1. Leong DP, Teo KK, Rangarajan S, Lopez-Jaramillo P, Avezum A, Jr., Orlandini A, et al. Prognostic value of grip strength: findings from the Prospective Urban Rural Epidemiology (PURE) study. Lancet. 2015;386(9990):266-73.

2. Silventoinen K, Magnusson PK, Tynelius P, Batty GD, Rasmussen F. Association of body size and muscle strength with incidence of coronary heart disease and cerebrovascular diseases: a populationbased cohort study of one million Swedish men. International journal of epidemiology. 2009;38(1):1108.

3. Ortega FB, Silventoinen K, Tynelius P, Rasmussen F. Muscular strength in male adolescents and premature death: cohort study of one million participants. Bmj. 2012;345:e7279.

4. Sayer AA, Syddall HE, Dennison EM, Martin HJ, Phillips DI, Cooper C, et al. Grip strength and the metabolic syndrome: findings from the Hertfordshire Cohort Study. QJM : monthly journal of the Association of Physicians. 2007;100(11):707-13.

5. Conroy RM, Pyorala K, Fitzgerald AP, Sans S, Menotti A, De Backer G, et al. Estimation of tenyear risk of fatal cardiovascular disease in Europe: the SCORE project. European heart journal. 2003;24(11):987-1003.

6. D'Agostino RB, Sr., Grundy S, Sullivan LM, Wilson P, Group CHDRP. Validation of the Framingham coronary heart disease prediction scores: results of a multiple ethnic groups investigation. Jama. 2001;286(2):180-7.

7. Assmann G, Schulte H, Cullen P, Seedorf U. Assessing risk of myocardial infarction and stroke: new data from the Prospective Cardiovascular Munster (PROCAM) study. European journal of clinical investigation. 2007;37(12):925-32.

8. Rantanen T, Harris T, Leveille SG, Visser M, Foley D, Masaki K, et al. Muscle strength and body mass index as long-term predictors of mortality in initially healthy men. The journals of gerontology Series A, Biological sciences and medical sciences. 2000;55(3):M168-73.

9. Firmann M, Mayor V, Vidal PM, Bochud M, Pecoud A, Hayoz D, et al. The CoLaus study: a population-based study to investigate the epidemiology and genetic determinants of cardiovascular risk factors and metabolic syndrome. BMC cardiovascular disorders. 2008;8:6.

10. Roberts HC, Denison HJ, Martin HJ, Patel HP, Syddall H, Cooper C, et al. A review of the measurement of grip strength in clinical and epidemiological studies: towards a standardised approach. Age and ageing. 2011;40(4):423-9.

11. Fried LP, Tangen CM, Walston J, Newman AB, Hirsch C, Gottdiener J, et al. Frailty in older adults: evidence for a phenotype. The journals of gerontology Series A, Biological sciences and medical sciences. 2001;56(3):M146-56.

12. Lean ME, Han TS, Morrison CE. Waist circumference as a measure for indicating need for weight management. Bmj. 1995;311(6998):158-61.

13. El Assaad MA, Topouchian JA, Darne BM, Asmar RG. Validation of the Omron HEM-907 device for blood pressure measurement. Blood pressure monitoring. 2002;7(4):237-41.

14. Moser M, Gencer B, Rodondi N. [Recommendations for management of dyslipidemia in 2014]. Revue medicale suisse. 2014;10(420):518, 20-4.

15. Nanchen D, Chiolero A, Cornuz J, Marques-Vidal PM, Firmann M, Mooser V, et al. Cardiovascular risk estimation and eligibility for statins in primary prevention comparing different strategies. The American journal of cardiology. 2009;103(8):1089-95.

16. Marrugat J, D'Agostino R, Sullivan L, Elosua R, Wilson P, Ordovas J, et al. An adaptation of the Framingham coronary heart disease risk function to European Mediterranean areas. Journal of epidemiology and community health. 2003;57(8):634-8. 
17. Marques-Vidal P, Rodondi N, Bochud M, Pecoud A, Hayoz D, Paccaud F, et al. Predictive accuracy and usefulness of calibration of the ESC SCORE in Switzerland. European journal of cardiovascular prevention and rehabilitation : official journal of the European Society of Cardiology, Working Groups on Epidemiology \& Prevention and Cardiac Rehabilitation and Exercise Physiology. 2008;15(4):402-8.

18. Marques-Vidal P, Rodondi N, Bochud M, Chiolero A, Pecoud A, Hayoz D, et al. Predictive accuracy of original and recalibrated Framingham risk score in the Swiss population. International journal of cardiology. 2009;133(3):346-53.

19. Canton de Vaud. Commission cantonale d'éthique de la recherche sur l'être humain. 2014. Available from: http://www.cer-vd.ch/. Accessed 5 January 2017.

20. Fujita Y, Nakamura Y, Hiraoka J, Kobayashi K, Sakata K, Nagai M, et al. Physical-strength tests and mortality among visitors to health-promotion centers in Japan. Journal of clinical epidemiology. 1995;48(11):1349-59.

21. Sasaki H, Kasagi F, Yamada M, Fujita S. Grip strength predicts cause-specific mortality in middleaged and elderly persons. The American journal of medicine. 2007;120(4):337-42.

22. Gale CR, Martyn CN, Cooper C, Sayer AA. Grip strength, body composition, and mortality. International journal of epidemiology. 2007;36(1):228-35.

23. Oppewal A, Hilgenkamp TI, van Wijck R, Evenhuis HM. The effect of handedness on grip strength in older adults with intellectual disabilities. Research in developmental disabilities. 2013;34(5):1623-9.

24. Petersen $\mathrm{P}$, Petrick $\mathrm{M}$, Connor $\mathrm{H}$, Conklin D. Grip strength and hand dominance: challenging the 10\% rule. Am J Occup Ther. 1989;43(7):444-7.

25. Incel NA, Ceceli E, Durukan PB, Erdem HR, Yorgancioglu ZR. Grip strength: effect of hand dominance. Singapore medical journal. 2002;43(5):234-7.

26. Kamarul T, Ahmad TS, Loh WY. Hand grip strength in the adult Malaysian population. Journal of orthopaedic surgery. 2006;14(2):172-7.

27. Zverev $Y$, Kamadyaapa D. Lateral asymmetry in grip strength: utility of the ten per cent rule. East African medical journal. 2001;78(11):611-5.

28. Osler M, Kriegbaum M, Christensen U, Lund R, Nybo Andersen AM. Loss to follow up did not bias associations between early life factors and adult depression. Journal of clinical epidemiology. 2008;61(9):958-63.

29. Rantanen T, Volpato S, Ferrucci L, Heikkinen E, Fried LP, Guralnik JM. Handgrip strength and cause-specific and total mortality in older disabled women: exploring the mechanism. Journal of the American Geriatrics Society. 2003;51(5):636-41.

30. Townsend N, Nichols M, Scarborough P, Rayner M. Cardiovascular disease in Europe 2015: epidemiological update. European heart journal. 2015;36(40):2673-4. 
Figure 1: Selection procedure. CoLaus Study, Lausanne, Switzerland, 2003-201206.

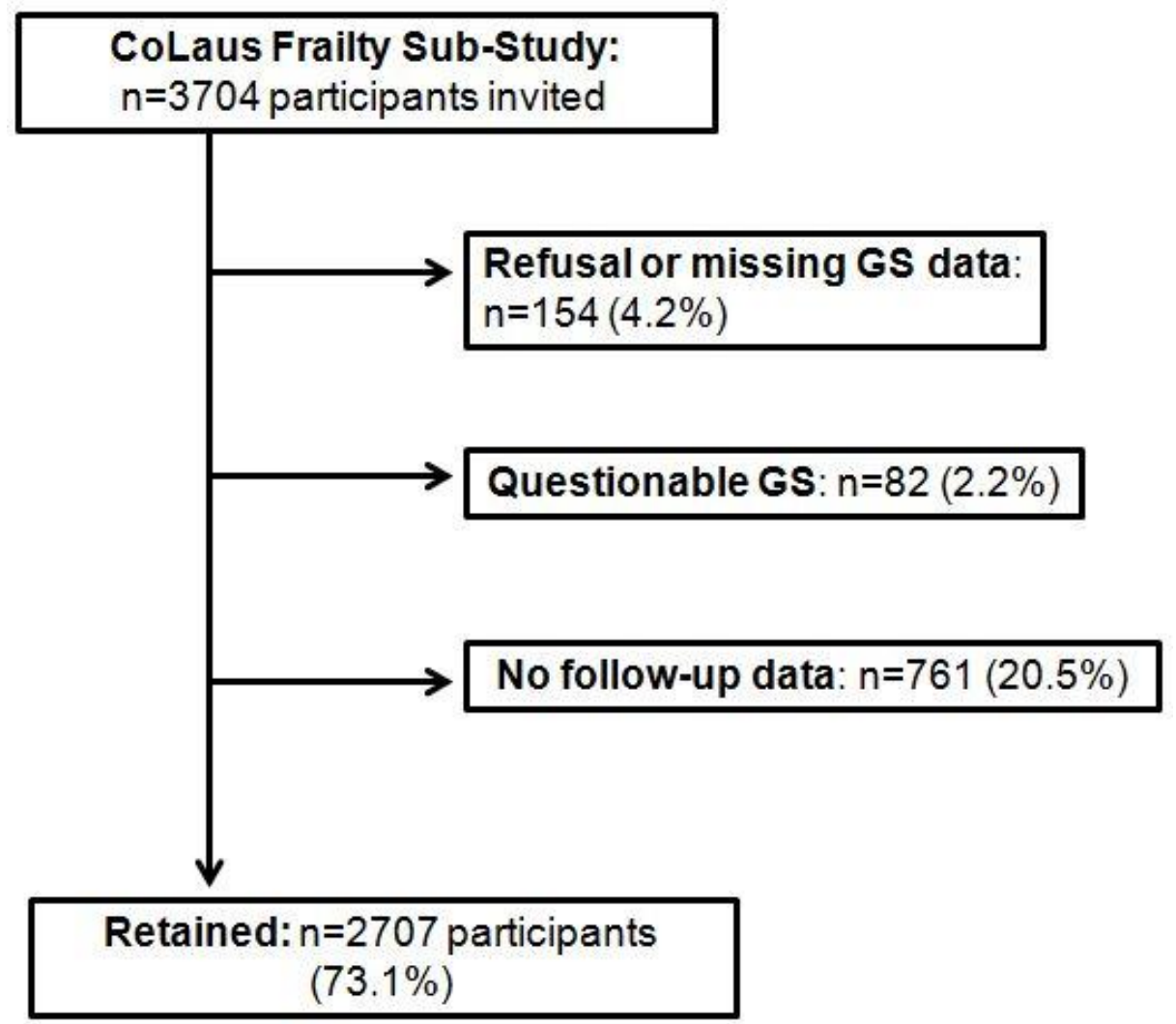

GS: grip strength. Percentages were calculated using the baseline sample size as denominator. 
Table 1: Characteristics of participants, overall and by grip strength categories. CoLaus Study, Lausanne, Switzerland, 2003-201206.

\begin{tabular}{|c|c|c|c|c|}
\hline & All & Normal & Low & P value \\
\hline $\mathrm{N}$ & 2707 & 2521 & 186 & \\
\hline Right-handedness (\%) & 92.0 & 91.9 & 93.2 & 0.52 \\
\hline Grip strength (kg) & $33.6 \pm 10.7$ & $34.5 \pm 10.5$ & $21.7 \pm 6.5$ & $<0.01$ \\
\hline Age (years) & $60.7 \pm 6.8$ & $60.4 \pm 6.7$ & $64.5 \pm 7.0$ & $<0.01$ \\
\hline Female (\%) & 54.8 & 55.0 & 51.6 & 0.37 \\
\hline Smoking (\%) & & & & 0.42 \\
\hline Current & 22.9 & 23.2 & 19.4 & \\
\hline Never & 39.1 & 38.8 & 42.5 & \\
\hline Former & 38.0 & 38.0 & 38.2 & \\
\hline Physical job (\%) & 15.2 & 15.2 & 14.1 & 0.67 \\
\hline Weekly leisure-time Pphysically activity & & & & $<0.01$ \\
\hline$<2$ periods of $20+$ minutes & 42.2 & 41.4 & 53.2 & \\
\hline$\geq 2$ periods of $20+$ minutes & 57.8 & 58.6 & 46.8 & \\
\hline Living alone (\%) & 35.1 & 34.9 & 38.2 & 0.37 \\
\hline Education level (\%) & & & & $<0.01$ \\
\hline Low & 58.5 & 57.7 & 69.4 & \\
\hline Middle & 24.5 & 24.9 & 19.4 & \\
\hline High & 17.0 & 17.4 & 11.3 & \\
\hline Job position (\%) & & & & $<0.01$ \\
\hline Low & 12.7 & 12.4 & 16.7 & \\
\hline Middle & 33.8 & 35.1 & 15.1 & \\
\hline High & 10.7 & 11.2 & 4.8 & \\
\hline Not working & 42.9 & 41.3 & 63.4 & \\
\hline Receiving social help (\%) & 30.0 & 28.1 & 55.4 & $<0.01$ \\
\hline Risk categories (SCORE) (\%) & & & & $<0.01$ \\
\hline Low & 41.3 & 42.6 & 24.3 & \\
\hline Medium & 14.3 & 14.4 & 12.4 & \\
\hline High & 16.7 & 17.1 & 11.9 & \\
\hline Very high & 27.7 & 25.9 & 51.4 & \\
\hline Risk categories (Framingham) (\%) & & & & $<0.01$ \\
\hline Low & 75.8 & 76.8 & 61.8 & \\
\hline Medium & 10.1 & 10.0 & 11.3 & \\
\hline High & 3.7 & 3.6 & 5.9 & \\
\hline Very high & 10.4 & 9.6 & 21.0 & \\
\hline Risk categories (PROCAM) (\%) & & & & $<0.01$ \\
\hline Low & 55.7 & 56.7 & 43.3 & \\
\hline Medium & 20.4 & 20.1 & 23.3 & \\
\hline High & 10.5 & 10.7 & 7.8 & \\
\hline Very high & 13.5 & 12.6 & 25.6 & \\
\hline
\end{tabular}

Results are expressed as mean \pm standard deviation or as percentage. Statistical analyses by chi-square or Student's t-tests comparing normal and low grip strength categories. 
Table 2: Association between low grip strength, overall mortality and incident cardiovascular events, unadjusted and multivariate-adjusted. CoLaus Study, Lausanne, Switzerland, 2003$2012 \theta 6$.

\begin{tabular}{lcccccc}
\hline & \multicolumn{3}{c}{ Overall mortality } & \multicolumn{3}{c}{ Incident cardiovascular events } \\
\hline Unadjusted & HR & {$[95 \%$ Cl] } & P value & HR & {$[95 \%$ CI] } & P value \\
Model 1 & 1.51 & $0.94-2.45$ & 0.09 & 1.76 & $1.13-2.76$ & 0.01 \\
Model 2 & 1.15 & $0.71-1.88$ & 0.57 & 1.22 & $0.78-1.93$ & 0.39 \\
Model 3 & 1.08 & $0.66-1.77$ & 0.75 & 1.07 & $0.68-1.70$ & 0.76 \\
Model 4 & 0.98 & $0.59-1.63$ & 0.95 & 0.96 & $0.60-1.55$ & 0.87 \\
Model 5 & 1.13 & $0.69-1.85$ & 0.62 & 1.23 & $0.79-1.94$ & 0.36 \\
Model 6 & 1.40 & $0.86-2.27$ & 0.17 & 1.34 & $0.86-2.10$ & 0.20 \\
\hline
\end{tabular}

Results are expressed as Hazard Ratio (HR) and 95\% confidence interval (Cl) for low grip strength using normal grip strength as the reference. Statistical analyses performed by Cox proportional hazard model, unadjusted and adjusted for: 1) age and gender; 2) age, gender, education level, job position and social help; 3) age, gender, education level, job position, social help, waist-to-hip ratio and height; 4) absolute CV risk according to SCORE risk equation; 5) absolute CV risk according to Framingham 2001 risk equation, and 6) absolute CV risk according to PROCAM 2007 risk equation. 
Table 3: power analyses for the results indicated in table 2. CoLaus Study, Lausanne, Switzerland, 2003-2012.

\begin{tabular}{lcccccc}
\hline & \multicolumn{3}{c}{ Overall mortality } & \multicolumn{3}{c}{ Incident cardiovascular events } \\
& Power & MSS & MDHR & Power & MSS & MDHR \\
\hline Unadjusted & 0.899 & 5,722 & 1.82 & 0.966 & 2,225 & 1.67 \\
Model 1 & 0.719 & 80,981 & 2.145 & 0.756 & 36,694 & 2.08 \\
Model 2 & 0.657 & 308,097 & 2.27 & 0.659 & 397,587 & 2.27 \\
Model Z 4 & 0.689 & 113,599 & 2.21 & 0.756 & 33,857 & 2.08 \\
Model 3 5 & 0.866 & 9,593 & 1.88 & 0.836 & 13,820 & 1.94 \\
Model 4 6 & 0.866 & 9,593 & 1.88 & 0.896 & 6,630 & 1.83 \\
\hline
\end{tabular}

Results are expressed as power to consider the observed $H R>1$ as statistically significant at $p=0.05$; the minimum sample size (MSS) to consider the observed $H R>1$ as statistically significant at a power of 0.80 and $p=0.05$, and the minimum detectable HR (MDHR) taking into account a sample size of 2707, 160 deaths and 188 incident CV events, a power of 0.80 and $p=0.05$. Calculations using the power cox function of Stata. Power analyses were not performed for model 3 as the observed HR were less than 1. 